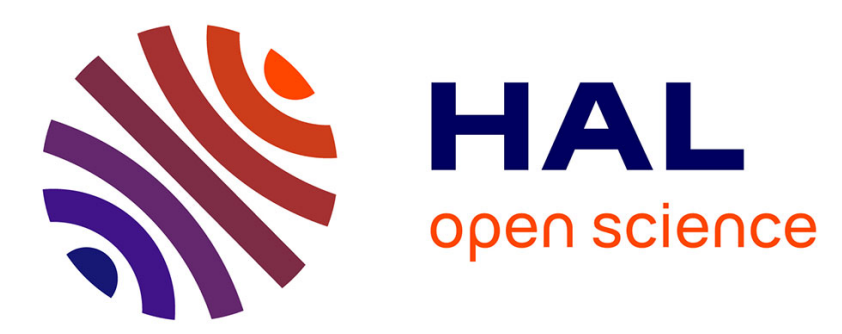

\title{
A numerical study of the hyperbolic manifolds in a priori unstable systems. A comparison with Melnikov approximations
}

\author{
Elena Lega, Massimiliano Guzzo, Claude Froeschlé
}

\section{- To cite this version:}

Elena Lega, Massimiliano Guzzo, Claude Froeschlé. A numerical study of the hyperbolic manifolds in a priori unstable systems. A comparison with Melnikov approximations. Celestial Mechanics and Dynamical Astronomy, 2010, 107 (1-2), pp.115-127. 10.1007/s10569-010-9265-7 . hal-00552507

\section{HAL Id: hal-00552507 https://hal.science/hal-00552507}

Submitted on 6 Jan 2011

HAL is a multi-disciplinary open access archive for the deposit and dissemination of scientific research documents, whether they are published or not. The documents may come from teaching and research institutions in France or abroad, or from public or private research centers.
L'archive ouverte pluridisciplinaire HAL, est destinée au dépôt et à la diffusion de documents scientifiques de niveau recherche, publiés ou non, émanant des établissements d'enseignement et de recherche français ou étrangers, des laboratoires publics ou privés. 


\title{
A numerical study of the hyperbolic manifolds in a priori unstable systems. A comparison with Melnikov approximations
}

\author{
Elena Lega · Massimiliano Guzzo • Claude Froeschlé
}

Received: 15 December 2009 / Revised: 5 February 2010 / Accepted: 26 February 2010 /

Published online: 6 April 2010

(C) Springer Science+Business Media B.V. 2010

\begin{abstract}
Using numerical methods we study the hyperbolic manifolds in a model of a priori unstable dynamical system. We compare the numerically computed manifolds with their analytic expression obtained with the Melnikov approximation. We find that, at small values of the perturbing parameter, the topology of the numerically computed stable and unstable manifolds is the same as in their Melnikov approximation. Increasing the value of the perturbing parameter, we find that the stable and unstable manifolds have a peculiar topological transition. We find that this transition occurs near those values of the perturbing parameter for which the error terms of Melnikov approximations have a sharp increment. The transition value is also correlated with a change in the behaviour of dynamical quantities, such as the largest Lyapunov exponent and the diffusion coefficient.
\end{abstract}

Keywords Hyperbolic chaos · FLI · Diffusion coefficient

\section{Introduction}

Stable and unstable manifolds are used since Poincaré to explain hyperbolic chaos and diffusion in dynamical systems. An important tool of analysis of these manifolds is the so-called Melnikov method (1963), which provides first order approximations of the manifolds in many situations. An important class of dynamical systems for which the Melnikov method works well is represented by the a priori unstable systems, following the terminology introduced in Chierchia and Gallavotti (1994), which consist in a small perturbation of an hyperbolic resonance. In absence of perturbation, the resonance has periodic hyperbolic orbits with their

E. Lega $(\varangle) \cdot$ C. Froeschlé

UNSA, CNRS UMR 6202, Observatoire de la Côte d'Azur,

B.P. 4229, 06304 Nice cedex 4, France

e-mail: elena@obs-nice.fr

M. Guzzo

Dipartimento di Matematica Pura ed Applicata, Università degli Studi di Padova, via Trieste 63, 35121 Padova, Italy 
stable and unstable manifold. The perturbation may change the topological properties of the stable and unstable manifolds in a way that may change also the dynamics in the resonance, introducing chaos and diffusion along the resonance. The mathematical tools to study the problem are of two kinds: perturbative or variational ones. This paper is concerned with the perturbative studies of stable and unstable manifolds, based on the Melnikov theory. Actually, already Poincare (1890) showed that the separatrices associated to integrable systems near simple resonances split under the effect of a perturbation. The Poincaré method was reconsidered seventy years later, by Melnikov (1963) and Arnold (1964), giving rise to the well known Poincaré-Melnikov-Arnold theory, more shortly addressed as Melnikov method. In the framework of a priori unstable systems, the Melnikov approximations have been used to prove the existence of homoclinic intersections among the stable and unstable manifolds, proving the existence of chaotic motions (as already argued by Poincaré 1890) and also to prove the existence of heteroclinic intersections among the stable and unstable manifolds, proving the existence of diffusion (see Arnold 1964' ${ }^{1}$; Chierchia and Gallavotti 1994; Treschev 2002, 2004). In the field of Celestial Mechanics the Melnikov method has been used for example for the study of capture orbits in the planar three body problem Easton (1991) or in the problem of escape of particles orbiting asteroids (Dankowicz 1997).

However, the perturbative nature of the Melnikov method does not allow one to understand what happens to the stable and unstable manifolds when increasing the perturbation parameter.

In this paper, using the Fast Lyapunov Indicator (introduced in Froeschlé et al. 1997 and deeply investigated in Guzzo et al. 2002), we compute numerically global representations of the stable and unstable manifolds in a model of a priori unstable system, allowing us to describe global properties of their topology. At small values of the perturbing parameter we find that the topology of the stable and unstable manifolds is the same as in their Melnikov approximation. Increasing the value of the perturbing parameter, we find that stable and unstable manifolds have a topological transition, and this topological transition occurs near the values of the perturbing parameter for which the Melnikov approximation is no more valid.

In a previous paper (Guzzo et al. 2009b), we have studied the Arnold diffusion for the same model of a priori unstable system. We have found that the behaviour of the diffusion coefficient $D$ with respect to the perturbing parameter is correlated to the validity of the Melnikov approximation. Precisely, the scaling law: $D(\epsilon) \simeq \epsilon^{2}$ is valid up to a critical value of $\epsilon$ for which the error terms of Melnikov approximations have a sharp increment. The result of Guzzo et al. (2009b) together with that of the present paper suggest that the Melnikov approximation is not only a technical tool which allows one to compute accurate approximations of the manifolds at small values of the perturbing parameters, but is related to a dynamical regime. This is confirmed by the computation of the largest Lyapunov exponents.

The paper is organized as follows: in Sect. 2 we define our model problem; in Sect. 3 we describe the numerical methods for detecting the structure of the stable (unstable) manifolds; in Sect. 4 we report the results on the computation of the global topology of the (un)stable manifolds. We connect the topology of the manifolds to the largest Lyapunov exponent and to the diffusion coefficient in 5. Conclusions are provided in 6.

\footnotetext{
1 The system studied in this paper is on the one hand quasi-integrable, because all harmonics are smaller than order $\epsilon$, and on the other hand is a priori unstable, because only one harmonic is indeed of order $\epsilon$ while the other ones are smaller than $\epsilon \mu$, with $\mu$ small parameter independent of $\epsilon$.
} 


\section{A model of a priori unstable system}

In this paper we consider the a priori unstable system introduced in Guzzo et al. (2009b) defined by the symplectic map:

$$
\begin{aligned}
\phi: \mathbb{T}^{4} & \longrightarrow \mathbb{T}^{4} \\
\left(\varphi_{1}, \varphi_{2}, I_{1}, I_{2}\right) & \longmapsto\left(\varphi_{1}^{\prime}, \varphi_{2}^{\prime}, I_{1}^{\prime}, I_{2}^{\prime}\right)
\end{aligned}
$$

such that:

$$
\begin{aligned}
& \varphi_{1}^{\prime}=\varphi_{1}+I_{1} \\
& \varphi_{2}^{\prime}=\varphi_{2}+I_{2} \\
& I_{1}^{\prime}=I_{1}-a \sin \varphi_{1}^{\prime}+\epsilon \frac{\sin \varphi_{1}^{\prime}}{\left(\cos \varphi_{1}^{\prime}+\cos \varphi_{2}^{\prime}+c\right)^{2}} \\
& I_{2}^{\prime}=I_{2}+\epsilon \frac{\sin \varphi_{2}^{\prime}}{\left(\cos \varphi_{1}^{\prime}+\cos \varphi_{2}^{\prime}+c\right)^{2}},
\end{aligned}
$$

where $a>0, \epsilon \geq 0$ and $c>2$ are parameters and $I_{1}, I_{2}, \varphi_{1}, \varphi_{2}$ are action-angle variables. The map $\phi$ has the following invariant manifold:

$$
\Lambda=\left\{\left(I_{1}, \varphi_{1}, I_{2}, \varphi_{2}\right): \text { such that }\left(I_{1}, \varphi_{1}\right)=(0, \pi)\right\}
$$

for any value of the parameters (for the notion of normally hyperbolic invariant manifolds see Hasselblatt and Pesin 2006; for the normal hyperbolicity of $\Lambda$ for the map (3) see Guzzo et al. 2009b). We just recall here that a manifold $\Lambda \subseteq \mathbb{T}^{4}$ is normally hyperbolic if, for any point $x \in \Lambda$, the tangent space $T_{x} \mathbb{T}^{4}$ has the following splitting:

$$
T_{x} \mathbb{T}^{4}=E^{s}(x) \oplus T_{x} \Lambda \oplus E^{u}(x)
$$

which is invariant; the so-called stable space $E^{s}(x)$ is contracting, the so-called unstable space $E^{u}(x)$ is expanding, and the contractions/expansions in $T_{x} \Lambda$ are less effective than the contractions/expansions in $E^{s}(x)$ and $E^{u}(x)$.

The importance of normally hyperbolic invariant manifolds is mainly stated by the so called local stable (unstable) manifold theorem, which states the existence, at any $x \in \Lambda$ of the smooth stable and unstable manifolds $W_{s}(x), W_{u}(x)$ of $x$ (see Hirsch et al. 1977), and of the stable and unstable manifolds of $\Lambda$ :

$$
W_{s}=\cup_{x \in \Lambda} W_{s}(x), \quad W_{u}=\cup_{x \in \Lambda} W_{u}(x) .
$$

For $\epsilon=0$ the map (3) is the product of two 2 dimensional uncoupled maps. The manifold $\Lambda$ is normally hyperbolic since its stable and unstable manifolds are the product of the stable and unstable manifolds of the hyperbolic fixed point $\left(I_{1}, \varphi_{1}\right)=(0, \pi)$ of the standard map:

$$
\varphi_{1}^{\prime}=\varphi_{1}+I_{1}, \quad I_{1}^{\prime}=I_{1}-a \sin \varphi_{1}^{\prime}
$$

with the torus $\mathbb{T}^{2}$, domain of $\left(I_{2}, \varphi_{2}\right)$. Because normal hyperbolicity persists for small perturbations, $\Lambda$ is normally hyperbolic also for suitably small $\epsilon$. In this case the stable and unstable manifolds are not a product as for $\epsilon=0$ and to describe their topology we will use Melnikov-like approximations and numerical techniques. By increasing $\epsilon$, the error terms of the Melnikov approximation becomes larger, and to compute the stable and unstable manifolds we use numerical methods. 
2.1 Detection of the phase-space structure of the model

In the last years the so called fast Lyapunov indicator (Froeschlé et al. 1997) has been extensively used to numerically detect the phase space structure, i.e. the distribution of KAM tori and resonances, of quasi-integrable systems (Froeschlé et al. 2000; Froeschlé and Lega 2000; Guzzo et al. 2002). For a map $\psi: M \rightarrow M$, the simplest definition of fast Lyapunov indicator of a point $x \in M$ and of a tangent vector $v \in T_{x} M$, at time $t$, is:

$$
\operatorname{FLI}_{t}(x, v)=\log \left(\frac{\left\|v_{t}\right\|}{\|v\|}\right)
$$

where $v_{t}=D \psi^{t}(x) v$. While the $\operatorname{limit}_{t \rightarrow \infty} \operatorname{FLI}_{t}(x, v) / t$ provides the Lyapunov exponent of the point $x$, the quantity $\operatorname{FLI}_{t}(x, v)$ provides informations about the dynamics already on the finite time $t$ : in the papers (Froeschlé et al. 2000; Froeschlé and Lega 2000; Guzzo et al. 2002) it is shown that, for quasi-integrable systems, if $t$ is suitably long (precisely of some inverse power of the perturbing parameter, see Guzzo et al. (2002) for precise statements and proofs) the value of $\operatorname{FLI}_{t}(x, v)$ is different, at order 0 in $\epsilon$, in the case $x$ belongs to an invariant KAM torus from the case $x$ belongs to a resonance of the system. Therefore, the computation of $\operatorname{FLI}_{t}(x, v)$ on grids of initial conditions $x \in M$ and a fixed vector $v$ allows one to detect the distribution of invariant tori and resonances in relatively short CPU times (Froeschlé et al. 2000; Froeschlé and Lega 2000).

Figure 1 shows the computation of the FLI for the map (3) for a grid of $500 \times 500$ initial conditions: $0<I_{1}<1,0<I_{2}<1, \varphi_{1}=\pi, \varphi_{2}=0$, for $t=1000$ iterations and different values of $\epsilon$ for $a=0.4$ and $c=2.1$
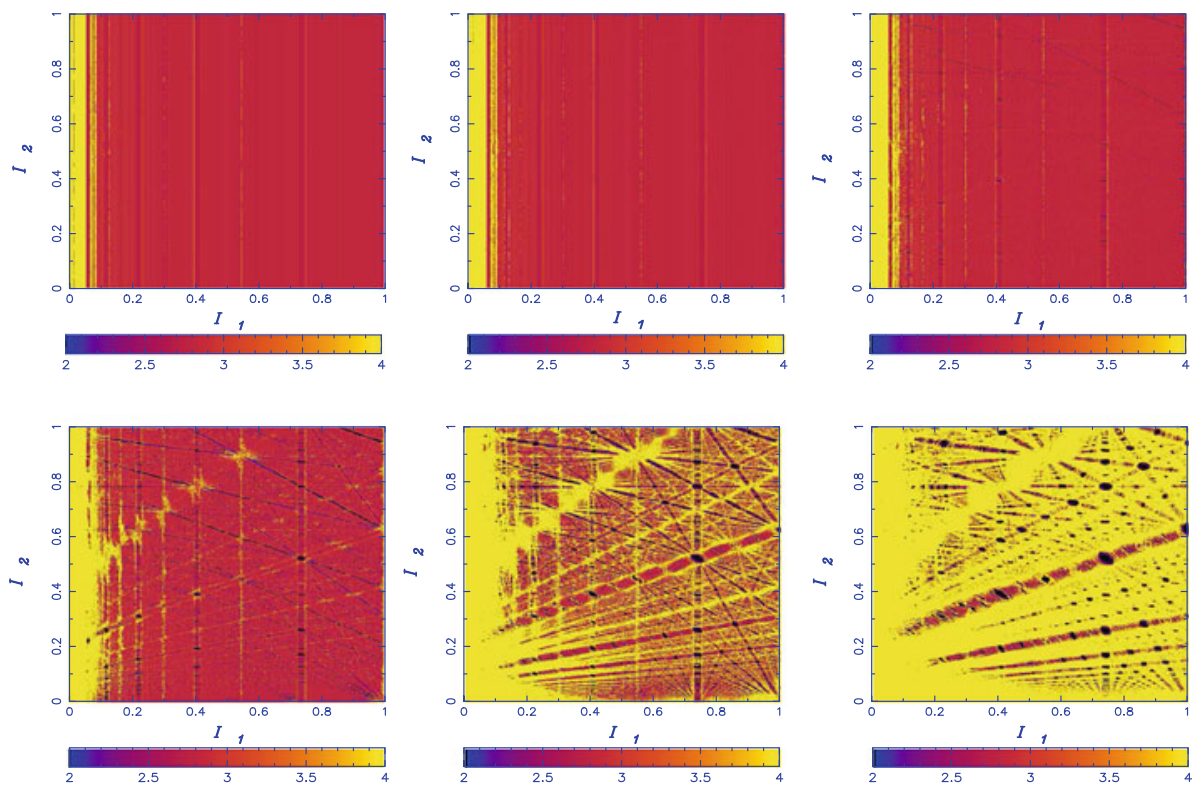

Fig. 1 FLI computation, for system (3) with $a=0.4, c=2.1$, on a grid of $500 \times 500$ initial conditions: $0<I_{1}<1,0<I_{2}<1, \varphi_{1}=\pi, \varphi_{2}=0$, for $t=1000$ iterations and different values of $\epsilon$. Top $\epsilon=0$, $\epsilon=6 \times 10^{-7}, \epsilon=6 \times 10^{-6}$. Bottom $\epsilon=6 \times 10^{-5}, \epsilon=3 \times 10^{-4}, \epsilon=6 \times 10^{-4}$. FLI $>4$ is represented by light gray color (darker grays correspond to lower values of the FLI. See color scale.) 
In the top left panel, $\epsilon=0$ we observe light gray vertical lines on a gray background. ${ }^{2}$ These lines correspond to the chaotic motions related of the standard map (6). The representation of the invariant manifold $\Lambda$ on this FLI-chart is the light grey, chaotic, line $I_{1}=0$. The grey background corresponds to the KAM tori of the standard map (6).

For $\epsilon=6 \times 10^{-7}$ (top, center panel of Fig. 1) the chart appears very similar to that for $\epsilon=0$ while increasing the perturbation to $\epsilon=6 \times 10^{-6}$ (top, right panel of Fig. 1) some secondary resonances appear on the picture.

Their effect increases by increasing $\epsilon$, as it is clear form the bottom figures. In particular, resonances overlap leaving only a small fraction of invariant tori remains for $\epsilon=3 \times 10^{-4}$ (bottom, center panel of Fig. 1), and the overlapping is complete for $\epsilon=6 \times 10^{-4}$ (bottom, right panel of Fig. 1). Let us remark that the above transition to Chirikov's overlapping of resonances is due to the interplay of the different resonances, and therefore it is better observed when the perturbation has already all harmonics at order $\epsilon$ as in (3). We also observe that for different values of $a$ and $c$, the critical values of $\epsilon$ change. The parameter $a$ determines directly the Lyapunov time of the standard map and the amplitude of its chaotic zone, but it is almost a scale parameter. The parameter $c$ changes more deeply the system: first, we must choose $c>2$ to avoid singularity of the perturbation. For increments of $c$ of order 1 nothing changes, except for the change of the critical parameters.

\subsection{The dynamics restricted to the normally hyperbolic invariant manifold}

Let us remark that the dynamics of $\phi$ defined in (3) restricted to $\Lambda$ is represented by the 2-dimensional map:

$$
\varphi_{2}^{\prime}=\varphi_{2}+I_{2}, \quad I_{2}^{\prime}=I_{2}+\epsilon \frac{\sin \varphi_{2}^{\prime}}{\left(\cos \varphi_{2}^{\prime}+c-1\right)^{2}},
$$

whose invariant KAM curves exclude any possibility of diffusion for $I_{2}$ if $\epsilon$ is suitably small. Let us fix an interval $D$ of the action $I_{2}$ and denote by $\epsilon_{c}$ the value such that the KAM theorem is valid in a open domain containing $D \times \mathbb{T}$ for any $0 \leq \epsilon \leq \epsilon_{c}$. From the analysis of numerically computed phase portraits of (8), with $c=2.1$, in the interval $I_{2} \in D=[0.26,0.38]$ we obtain that for $0 \leq \epsilon \leq 0.002$ (Fig. 2 left panel for $\epsilon=0.002$ ) the map has still many invariant tori which constitute a topological barrier to the diffusion of the action $I_{2}$. Instead, for $\epsilon=0.0026$ the invariant tori seem to have disappeared (Fig. 2, right panel), leaving the possibility of chaotic diffusion in the direction of the action $I_{2}$. Therefore, this is a numerical indication that $\epsilon_{c} \in(0.002,0.0026)$.

Actually, as soon as $\epsilon$ is bigger than $\epsilon_{c}$, diffusion of the action $I_{2}$ is possible, but it can be very slow because of possible stickiness phenomena (see, for example, Efthymiopoulos et al. 1999) due to the presence of cantori and islands of regular motion MacKay et al. (1984). These barriers to diffusion loose their effectiveness at higher values of $\epsilon$. The Arnold diffusion for the map (3) in the neighborhood of $\Lambda$ was studied in Guzzo et al. (2009b). In this paper we investigate numerically the topological properties of the (un)stable manifolds of $\Lambda$ and we compare them to the dynamical properties of a neighborhood of $\Lambda$.

2 The color version of all figures can be found on the electronic version of the paper so that light gray corresponds there to yellow and darker gray corresponds there to darker orange. 

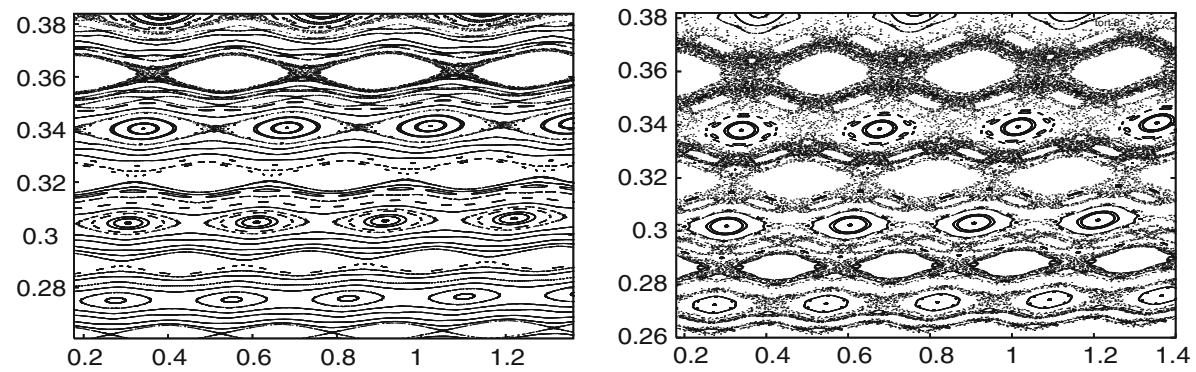

Fig. 2 Phase portrait of the restricted map (8) for $\epsilon=0.002,0.0026$ and $c=2.1$

\section{Numerical detection of the stable and unstable manifolds}

In this section we describe a recent application of the FLI method to detect numerically the global structure of the stable and unstable manifolds, precisely of their intersection with any two dimensional surface of the phase-space. We have recently shown that, looking at proper resolution, the FLI reveals any detail of the structure of stable and unstable manifolds (Guzzo 2007, 2010; Guzzo et al. 2009a). A detailed application of the FLI to the detection of the stable-unstable manifolds in relation with space flight dynamics has been done by Villac (2008). We provide below an explanation. We sample two dimensional surfaces of the phase space with grids of points, and for any point $x$ of the grid and the same vector $v$ we compute the $\operatorname{FLI}_{T}(x, v)$ for some time $T$. The points of the grid which have the highest values of the FLI are those points whose orbits approach an hyperbolic invariant manifold within the time $T$, because the growth of tangent vectors is bigger near the hyperbolic manifolds. Therefore, a short-time computation of the FLI allows one to detect a neighborhood of a finite piece of the stable manifold (the unstable manifold can be obtained by computing the FLI of the inverse map). As a check on a well known example, we compare in Fig. 3 the computation of finite pieces of the unstable manifold of the hyperbolic fixed point of the standard map:

$$
\varphi^{\prime}=\varphi+I, \quad I^{\prime}=I-a \sin \varphi^{\prime}
$$

with $a=0.4$. In the left panel we report the computation of finite pieces of the unstable manifold in a neighborhood of the fixed point obtained by using the traditional method of propagation of sets of points (described, for example, in Simó 1989). In the right panel we report the computation of the FLI of the inverse map for a grid of points in the same neighborhood of the fixed point for $T=40$ iterations of the map. The light gray lines in the right panel represent the points of the grid with the highest values of the FLI, and they clearly correspond to pieces of the unstable manifold. At difference with Fig. 1, all the motions analyzed here are chaotic.

The agreement among the results of the two methods is good and in particular we remark the sharpness of the detection of the stable manifold with the FLI method. The application of the FLI method to higher dimensional systems is nearly as simple as in this two dimensional case, and one does not need to know in advance which are the normally hyperbolic invariant manifolds and their local approximations. In fact, the method detects the stable manifolds of all the hyperbolic structures of the system.

The application of this method to the detection of stable manifolds in more complicate examples, such as the map (3), is described in Sect. 4. 

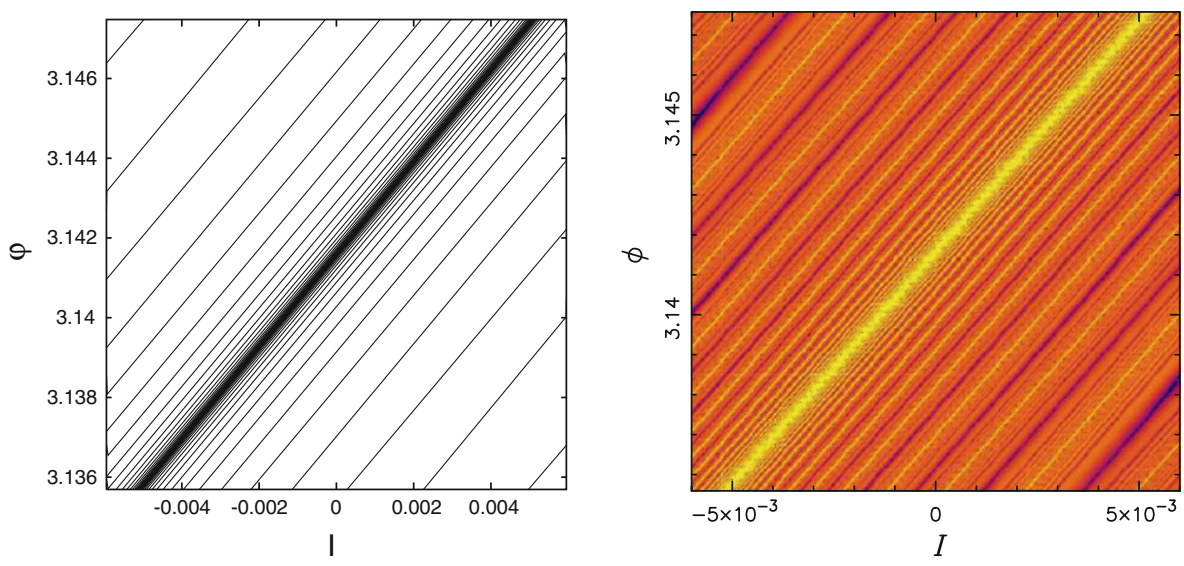

Fig. 3 Detection of a piece of the unstable manifold of the standard map (9) in the neighborhood of the hyperbolic fixed point $\varphi \in[\pi-0.006, \pi+0.006], I \in[-0.006,0.006]$. On the left detection of the manifold with the traditional method of propagation of sets. On the right detection of the manifold with the FLI method. The FLI has been computed using the inverse of the standard map (9), a set of $500 \times 500$ initial conditions, a maximum of $T=40$ iterations of the map. The color scale range is such that FLI $>3.3$ is represented by light gray color (darker grays correspond to lower values of the FLI). All the motions are chaotic

\section{Topology of the stable and unstable manifolds}

In this section we compute different representations of the stable and unstable manifolds of $\Lambda$ for $a>0$. When $\epsilon=0$ the stable and unstable manifolds $W_{s}, W_{u}$ of $\Lambda$ are the product of the stable and unstable manifolds $W_{s}^{*}, W_{u}^{*}$ of the hyperbolic fixed point of the standard map (6) with the torus $\mathbb{T}^{2}$, domain of $\left(\varphi_{2}, I_{2}\right)$. To provide a representation of $W_{s}, W_{u}$, we compute their intersection with the two dimensional surface of the phase-space:

$$
S=\left\{\left(\varphi_{1}, \varphi_{2}, I_{1}, I_{2}\right) \text { such that }: \varphi_{1}=\pi, \varphi_{2}=0\right\},
$$

that is we compute the sets:

$$
S_{u}^{*}=S \cap W_{u}, \quad S_{s}^{*}=S \cap W_{s} .
$$

Actually, the computation can be done for all possible sections. However, for a clear interpretation of the results, we find that the section must intersect $\Lambda$, so that we can appreciate what happens to initial conditions closer and closer to the manifold. Therefore $\varphi_{1}=\pi$ is a mandatory choice. Instead, $\varphi_{2}$ can be freely chosen, and in this case we did not observe qualitative changes to the figures reported in the paper. The reason is that $\varphi_{2}$ parameterize the invariant tori of $\Lambda$, and therefore a different choice means only a approaching $\Lambda$ in a different point of these tori.

When $\epsilon=0$ it is:

$$
S_{u}^{*}=\left\{\left(\varphi_{1}, \varphi_{2}, I_{1}, I_{2}\right) \text { such that }: \varphi_{1}=\pi,\left(I_{1}, \varphi_{1}\right) \in W_{u}^{*}, \varphi_{2}=0\right\},
$$

that is a set of lines parallel to the $I_{2}$ axis with accumulations. The same holds for $S_{s}^{*}$. When $\epsilon \neq 0$ understanding the topology of $S_{u}^{*}, S_{s}^{*}$ becomes a difficult problem, but if $\epsilon$ is very small, we can use the Melnikov like approximations introduced in Guzzo et al. (2009b). Following Guzzo et al. (2009b), we define the Melnikov approximation of $W_{u}(x)$ as the unstable manifold of $x$ with respect to the following simplified map $\tilde{\phi}$ : 


$$
\begin{array}{ll}
\varphi_{1}^{\prime}=\varphi_{1}+I_{1} & \varphi_{2}^{\prime}=\varphi_{2}+J \\
I_{1}^{\prime}=I_{1}-a \sin \varphi_{1}^{\prime} & I_{2}^{\prime}=I_{2}+\epsilon \frac{\sin \varphi_{2}^{\prime}}{\left(\cos \varphi_{1}^{\prime}+\cos \varphi_{2}^{\prime}+c\right)^{2}} .
\end{array}
$$

where $J$ denotes the $I_{2}$ component of $x$. Let us remark that usually the Melnikov method (Arnold 1964, and more recently Chierchia and Gallavotti 1994; Treschev 2002, 2004) is applied to continuous systems and approximations of stable and unstable manifolds are obtained as integrals. In Guzzo et al. (2009b), we have provided the explicit representation of the approximated manifold as a series of a function computed over an orbit of the unperturbed system (with $\epsilon=0$ ). Moreover, for $\epsilon=0$, the stable and unstable manifolds of $\Lambda$ already intersect transversely, because the unperturbed system is the standard map coupled with a rotation. This is different from Arnold (1964), Chierchia and Gallavotti (1994), Treschev (2002) and Treschev (2004), where unperturbed systems are hyperbolic, but without transverse intersections of the manifolds. If $\left(\tilde{I}_{1}, \tilde{\varphi}_{1}, \tilde{I}_{2}, \tilde{\varphi}_{2}\right)$ is in the Melnikov approximation of $W_{u}(x)$, then $\left(\tilde{I}_{1}, \tilde{\varphi}_{1}\right) \in W_{u}^{*}$, and this condition is independent of $I_{2}$. As a consequence, the Melnikov approximation of $S_{u}^{*}$ is a set of lines parallel to the $I_{2}$ axis with accumulations, as in the unperturbed case.

\subsection{A transition in the topology of $S_{s}^{*}, S_{u}^{*}$}

We considered the map (3) with $c=2.1, a=0.4$ and we computed $S_{s}^{*}$ for different values of $\epsilon$ by computing the FLI on refined grids of $1000 \times 1000$ regularly spaced points of $S$. The results are shown in Figs. 4 and 5: the three columns of the figures represent different zooms of $S$ with respect to the action $I_{1}$, allowing one to appreciate the topology of $S_{s}^{*}$ from the small values of $I_{1} \in\left[10^{-11}, 10^{-8}\right]$ (left column) up to $I_{1}$ of order 0.1 (right column). Each line refers to a different value of $\epsilon$, so that we can appreciate the evolution of $S_{s}^{*}$ from $\epsilon=0$ up to $\epsilon$ of order $10^{-3}$. The action $I_{2}$ is in the range $[0,1]$. We now comment the results. For $\epsilon=0$ (top line of Fig. 4) we recognize that $S_{s}^{*}$ is a set of lines parallel to the axis $I_{1}=0$ with accumulation towards $I_{1}=0$, as we expected. For $\epsilon=10^{-6}$ (second line of Fig. 4) the situation is very similar to the case with $\epsilon=0: S_{s}^{*}$ seems to be represented by vertical lines (of course with small deviations), as it is expected if the Melnikov approximation is valid. For $\epsilon=6 \times 10^{-6}$ (last line of Fig. 4) most of the vertical lines are still visible in the three zooms, though with an evident distortion. However, the vertical lines have disappeared in some regions. For $\epsilon=4 \times 10^{-5}$ (top line of Fig. 5) we are close to a transition in the topology of all vertical lines, which becomes more evident for $\epsilon=6 \times 10^{-5}$ (second line of Fig. 5), where horizontal lines appear. This kind of topology cannot be explained by the Melnikov approximation, which is therefore not valid for this value of $\epsilon$. Let us remark that $S_{u}^{*}$ is a set of lines parallel to the $I_{2}$ axis with accumulation both in the unperturbed case and in the Melnikov approximation, therefore one could say that for $\epsilon=6 \times 10^{-5}$ the topology simply changes with respect to the unperturbed system. However, when computing, as in Guzzo et al. (2009b), the unstable manifold $\tilde{W}_{u}(x)$ of a point $x$ of $\Lambda$ with the mapping $\tilde{\phi}$ it turns out to be different from the unstable manifold of a point of $\Lambda$ of the unperturbed system since $I_{2}$ is not constant for $\epsilon \neq 0$. Therefore, the comparison between $W_{u}(x)$ and $\tilde{W}_{u}(x)$ provides a way to compute the threshold of validity of the Melnikov approximation, which turns out to be (see Guzzo et al. 2009b) almost the same as the threshold of transitions of the global manifold.

For $\epsilon=6 \times 10^{-4}$ (last line of Fig. 5) the transition in the topology of $S_{S}^{*}$ is complete: the inner zoom shows only horizontal lines and also the outer zoom reveals a topology which is completely different from the one which is expected in the Melnikov approximation. We 

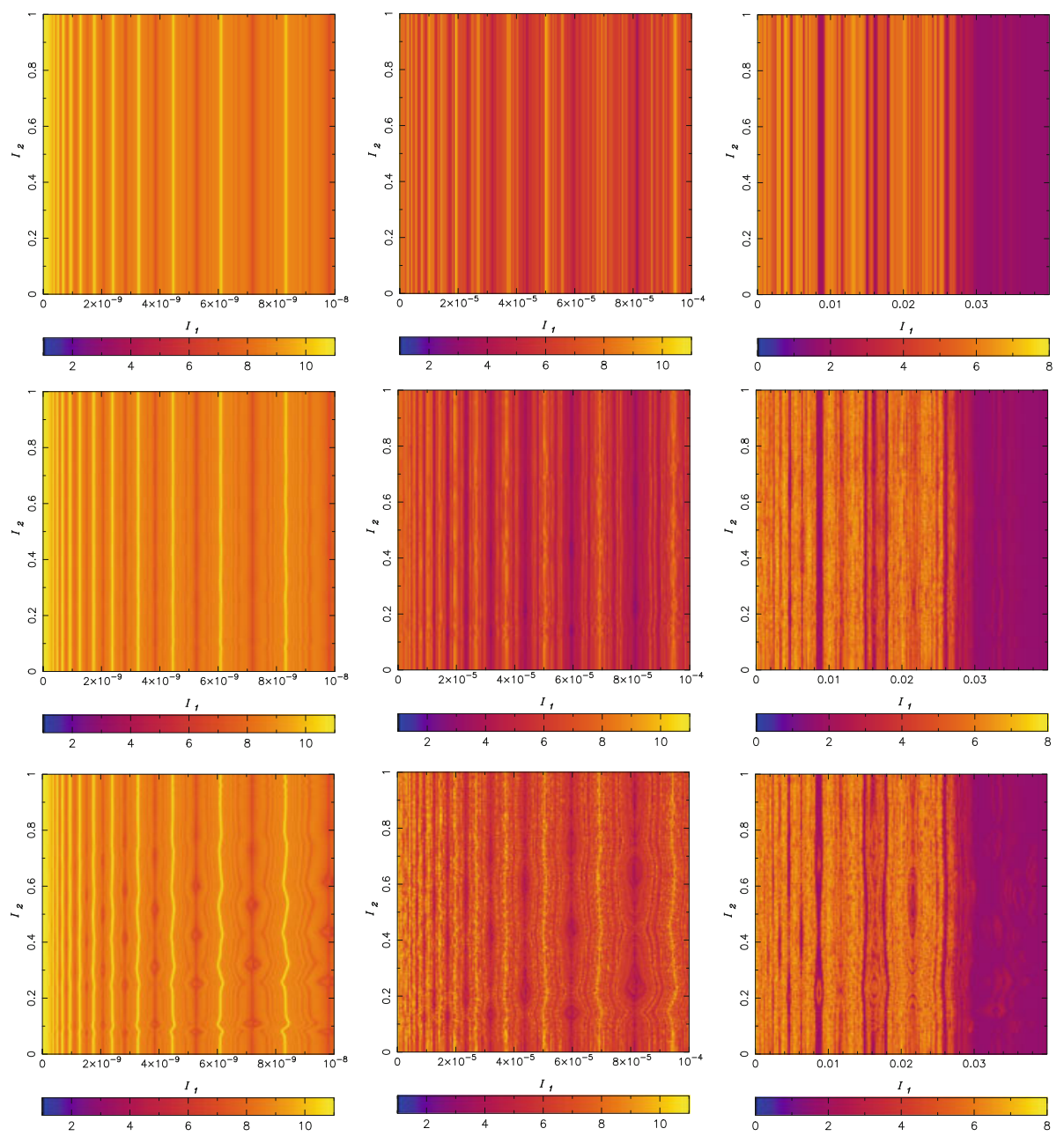

Fig. 4 Computation of $S_{s}^{*}$ for $I_{1}<10^{-8}\left(t=60\right.$, left panels), for $I_{1}<10^{-4}(t=80$, middle panels $)$ and $I_{1}<4 \times 10^{-2}\left(t=80\right.$, right panels). The perturbation is (from top to bottom) $\epsilon=0,10^{-6}, 6 \times 10^{-6}$. The light-gray lines correspond to finite pieces of the stable manifold

say that for this value of $\epsilon$ the transition of the topology of $S_{s}^{*}$ in the range of $I_{2} \in[0,1]$ is completed.

We repeated these computations for $I_{2} \in[1.4,2.4]$, and we detected the same kind of transitions in the topology of $S_{s}^{*}$. The topological transition is due to the resonances of $\Lambda$. Increasing $\epsilon$ the size of such resonances increases and drastically changes the global topology of $W_{u}$.

Summarizing these results, we have shown that for small values of $\epsilon$ the topology of $S_{s}^{*}$ is consistent with the description of the stable (unstable) manifolds obtained with the Melnikov approximation, i.e. it is characterized by the prevalence of vertical lines. For high values of $\epsilon$ the topology is characterized by horizontal lines which originate at the resonances on $\Lambda$. This happens even for values of $\epsilon$ such that the restricted map has still a lot of invariant tori, so that the transition in the topology of $S_{s}^{*}$ is not related to the transition to the 

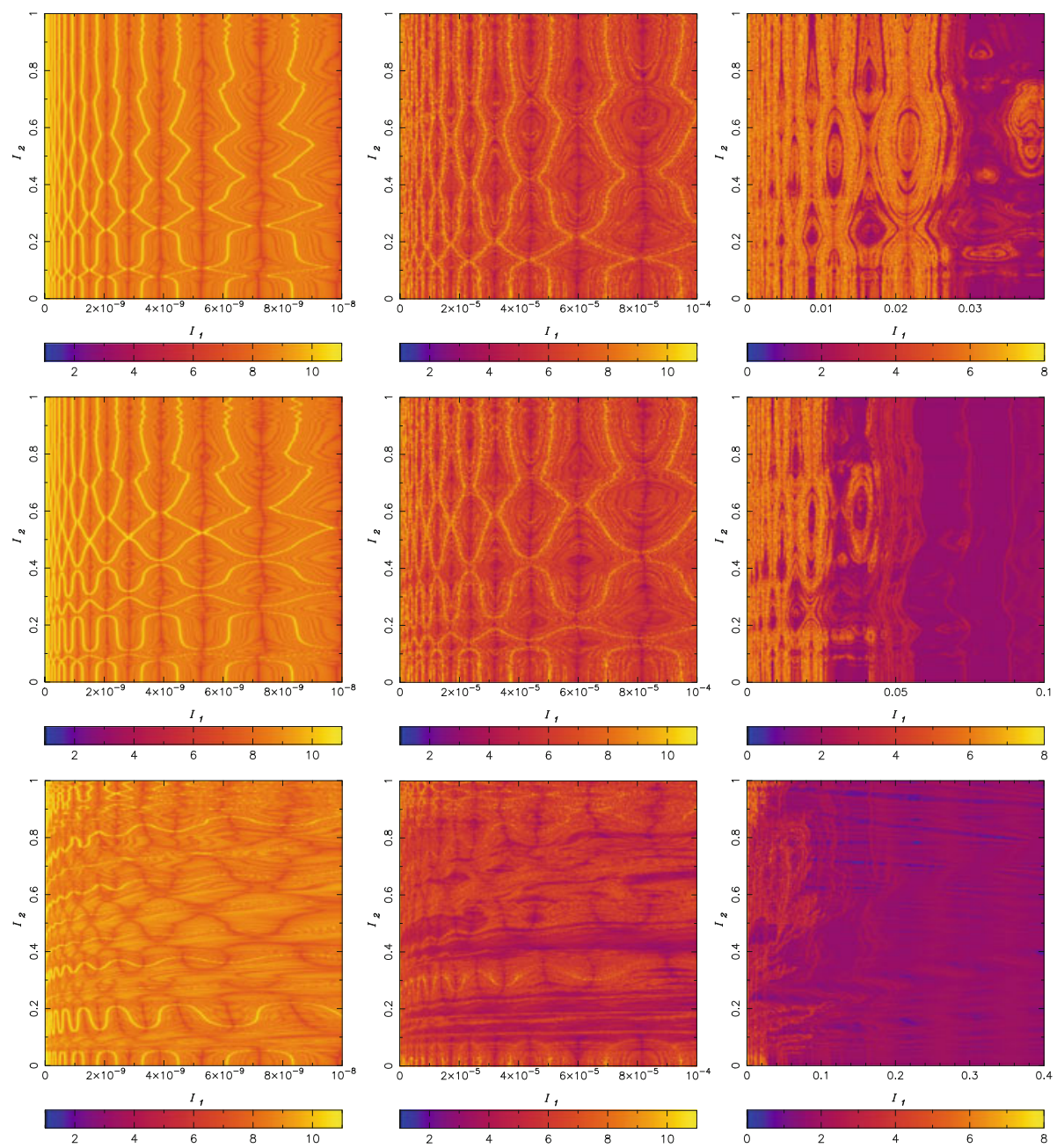

Fig. 5 Computation of $S_{s}^{*}$ for $I_{1}<10^{-8}(t=60$, left panels $)$, for $I_{1}<10^{-4}(t=80$, middle panels $)$ and $I_{1}<$ $4 \times 10^{-1}\left(t=80\right.$, right panels). The perturbation is (from top to bottom) $\epsilon=4 \times 10^{-5}, 6 \times 10^{-5}, 6 \times 10^{-4}$. The light-gray lines correspond to finite pieces of the stable manifold

Chirikov regime of $\phi_{\mid \Lambda}$. For intermediate values of $\epsilon$ we detect a transition among the two topologies, in which the vertical lines are distorted up to be completely replaced by horizontal lines.

\section{On the connection between topology and dynamical indicators}

In order to better investigate the transition in the topology and its correlations with the dynamics, in this section we report the computation of two dynamical indicators, such as the Lyapunov Characteristic Indicator and the diffusion coefficient, for many values of $\epsilon$.

The Lyapunov Characteristic Indicator has been computed for $\epsilon \in\left[10^{-10}, 10^{-2}\right]$. In Fig. 6 we report the mean value and the standard deviation of the LCI computed for 
Fig. 6 Mean value and standard deviation of the LCI as a function of $\epsilon$ for two sets of 100 orbits with initial conditions in a neighborhood of two different hyperbolic tori of $\Lambda$. The initial conditions: $-10^{-6}<I_{1}(0)<$ $10^{-6}, \varphi_{1}(0)=\pi, \varphi_{2}(0)=0$ and, respectively $I_{2}(0)=0.324$ and $I_{2}(0)=1.8$

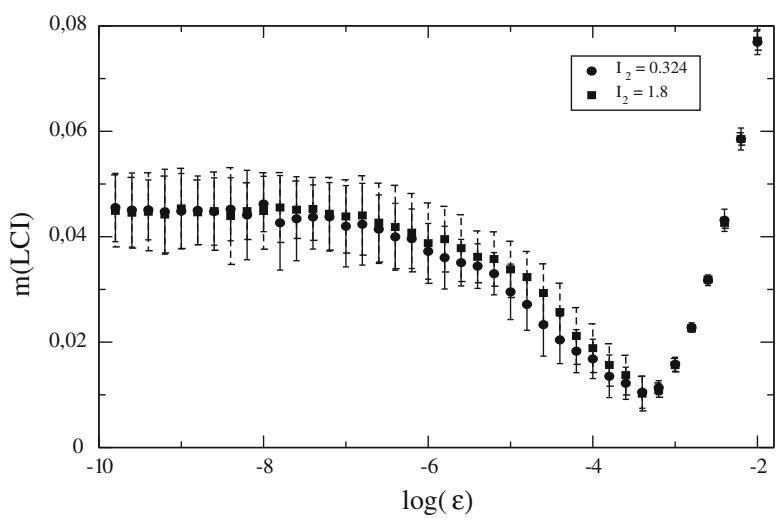

$10^{8}$ iterations over two sets of 100 orbits with initial conditions on the resonance $I_{1}=0$ : $-10^{-6}<I_{1}(0)<10^{-6}, \varphi_{1}(0)=\pi, \varphi_{2}(0)=0$ and, respectively $I_{2}(0)=0.324$ and $I_{2}(0)=1.8$ The LCI appears to be constant for both sets up to $\epsilon \simeq 5 \times 10^{-6}$. The strength of chaos for very small values of $\epsilon$ depends mainly on the parameter $a$ thus explaining the constant LCI value. For $5 \times 10^{-6}<\epsilon<3 \times 10^{-4}$, we observe a change of behavior in the LCI data. For $\epsilon>3 \times 10^{-4}$ the LCI starts to increase and the standard deviation decreases. This is the signature that ergodicity is reached within the integration time. This is typical of the Chirikov's regime of overlapping of resonances which actually starts close to $\epsilon \simeq 3 \times 10^{-4}$ (Fig. 1, bottom center). The interval $5 \times 10^{-6}<\epsilon<3 \times 10^{-4}$ is in agreement with the interval of transition in the topology of the normally hyperbolic invariant manifold $\Lambda$.

The diffusion coefficient has been computed numerically with the techniques which we used in Froeschlé et al. (2000), Lega et al. (2003), Guzzo et al. (2005), Froeschlé et al. (2005) and Guzzo et al. (2006).

Precisely, we measured the diffusion coefficient for $a=0.4, c=2.1$ for different values of $\epsilon$ for two sets of $N=100$ initial conditions with $I_{2}=0.324, I_{2}=1.8$, respectively (the other initial conditions are $\left.I_{1} \in\left[-10^{-6}, 10^{-6}\right], \varphi_{1}=\pi, \varphi_{2}=0\right)$. The average evolution of the mean squared distance of the action $I_{2}$ from its initial value grows almost linearly with time for most values of $\epsilon$, the slope giving the diffusion coefficient $D$. The linear law is verified by means of a $\chi$-square fit with correlation coefficient which turns out to be lower than $97 \%$ in the interval $5 \times 10^{-6}<\epsilon<5 \times 10^{-4}$ with a minimum value of $92 \%$ for $\epsilon=1.5 \times 10^{-5}$ for the set of data with initial conditions centered on $I_{2}=1.8$. Let us remark that in Guzzo et al. (2009b) we used a more strict criterium to define the 'good' statistics. The statistics turns out to be less good for $\epsilon>10^{-6}$, i.e. some irregularity appear in the diffusion curve because of a more irregular behaviour in the diffusion of the orbits. Therefore, as the irregularities of the frequency map allow one to use frequency analysis to detect the presence of a resonance, we use the irregularity of the diffusion coefficient (and of its confidence) to detect a change in the dynamics.

The results of the computation are reported in Fig. 7. For the two sets of initial conditions the dependence of the diffusion coefficient on $\epsilon$ is well fitted by a power law $D(\epsilon) \simeq \epsilon^{2}$ for $\epsilon \leq 6 \times 10^{-6}$. In this case the heuristic (because we do not have a proof of it, while proofs exist for a continuous priori unstable systems) diffusion argument provided by the Arnold mechanism of diffusion suggests that the diffusing orbits follow closely pieces of the stable and unstable manifolds $W_{s, u}(x)$ of points $x$ of $\Lambda$. In this situations, the manifolds $W_{s, u}(x)$ and their splitting angles are computed using Melnikov approximations, as it was done in 
Fig. 7 Variation of the diffusion coefficient as a function of $\epsilon$. Data are very well fitted to a power law $D(\epsilon) \simeq \epsilon^{2}$ for $10^{-10} \leq \epsilon \leq 6 \times 10^{-6}$. For $6 \times 10^{-6} \leq \epsilon \leq 4 \times 10^{-4}$ some irregularity can appear depending on the choice of initial conditions, although data are not far from the $D(\epsilon) \simeq \epsilon^{2}$ law

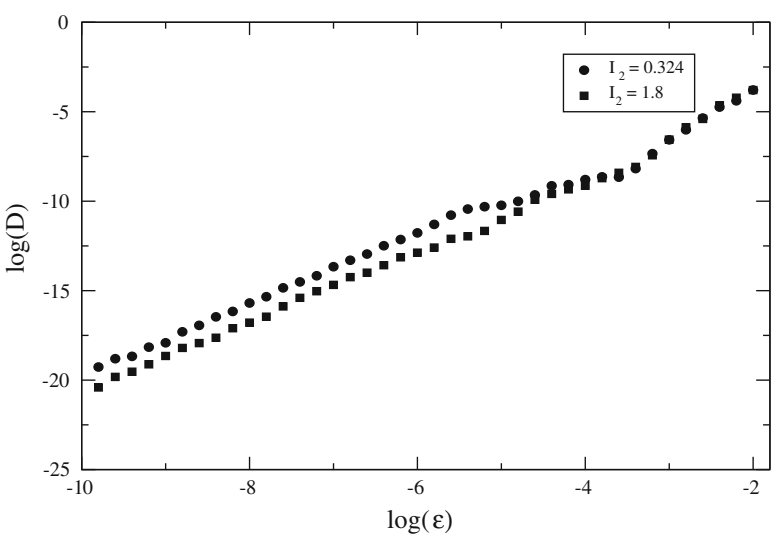

Arnold (1964), Chierchia and Gallavotti (1994), Treschev (2002) and Treschev (2004). In Guzzo et al. (2009b) we checked that $\tilde{W}_{s, u}(x)$ fits correctly $W_{s, u}(x)$ up to $\epsilon \leq 6 \times 10^{-6}$ while for larger values of $\epsilon$ the Melnikov approximation loses its validity.

For $6 \times 10^{-6} \leq \epsilon \leq 4 \times 10^{-4}$ some irregularity can appear depending on the specific set of initial conditions, although data are not far from the $D(\epsilon) \simeq \epsilon^{2}$ law. At this transition it occurs also a transition of the global stable and unstable manifolds. The topological properties of this transition cannot be appreciated by the computation of the single $W_{s, u}(x)$. For $\epsilon$ larger than the transition value, the global manifolds have a topology which is completely different from the first orders approximations, and the diffusion coefficient depends irregularly on $\epsilon$. In this situation we guess that the system is outside the range of application of perturbation theories, and therefore we are not able to provide explanations. Maybe, the variational approaches, which are successfully used for high values of perturbing parameters, may provide an explanation, but this is outside the goals of this paper.

For $\epsilon>4 \times 10^{-4}$, i.e in the overlapping of resonances regime, the power law changes to $D(\epsilon) \simeq \epsilon^{2.8}$.

\section{Conclusions}

In this paper we have numerically detected the topology of the hyperbolic manifolds supporting diffusion in the a priori unstable dynamical systems. Using the Melnikov-like approximation of the unstable manifolds introduced in Guzzo et al. (2009b) we have shown that stable and unstable manifolds have a topological transition when the Melnikov approximation looses its accuracy. This transition is correlated to a change of the law of dependence of the diffusion coefficient on the perturbing parameter as well as to a change of behaviour of the largest Lyapunov exponent. This suggests that the Melnikov approximation is not only a technical tool which allows one to compute accurate approximations of the manifolds at small values of the perturbing parameters, but is related to a dynamical regime.

\section{References}

Arnold, V.I.: Instability of dynamical systems with several degrees of freedom. Sov. Math. Dokl. 6, 581585 (1964)

Chierchia, L., Gallavotti, G.: Drift and diffusion in phase space. Ann. Inst. H.Poincaré 60, 1-144 (1994) 
Dankowicz, H.: Escape of particle orbiting asteroids in the presence of radiation pressure through separatrix splitting. Celest. Mech. Dyn. Astron. 67, 63-85 (1997)

Easton, R.W.: Capture orbits and Melnikov integrals in the planar three-body problem. Celest. Mech. Dyn. Astron. 50, 283-297 (1991)

Efthymiopoulos, C., Contopoulos, G., Voglis, N.: Cantori, islands and asymptotic curves in the stickiness region. Celest. Mech. Dyn. Astron. 73(1/4), 221-230 (1999)

Froeschlé, C., Lega, E., Gonczi, R.: Fast Lyapunov indicators. Application to asteroidal motion. Celest. Mech. Dyn. Astron. 67, 41-62 (1997)

Froeschlé, C., Guzzo, M., Lega, E.: Graphical evolution of the Arnold Web: from order to Chaos. Science 289 (5487), 2108-2110 (2000)

Froeschlé, C., Lega, E.: On the structure of symplectic mappings. The fast Lyapunov indicator: a very sensitive tool. Celest. Mech. Dyn. Astron. 78, 167-195 (2000)

Froeschlé, C., Guzzo, M., Lega, E.: Local and global diffusion along resonant lines in discrete quasi-integrable dynamical systems. Celest. Mech. Dyn. Astron. 92, 243-255 (2005)

Guzzo, M., Lega, E., Froeschlé, C.: On the numerical detection of the effective stability of chaotic motions in quasi-integrable systems. Physica D 163, 1-25 (2002)

Guzzo, M., Lega, E., Froeschlé, C.: First numerical evidence of global Arnold diffusion in quasi-integrable systems. DCDS B 5, 687-698 (2005)

Guzzo, M., Lega, E., Froeschlé, C.: Diffusion and stability in perturbed non-convex integrable systems. Nonlinearity 19, 1049-1067 (2006)

Guzzo, M.: Mechanisms for the production of chaos in dynamical systems. In: Proceedings of 'Scottish Universities Summer Schools in Physics No. 62 Extra-Solar Planets: the detection, formation, evolution and dynamics of planetary systems' held in Skye in 28th May-8th June (2007). Accepted for publication

Guzzo, M.: Chaos and diffusion in dynamical systems through stable-unstable manifolds. In: Perozzi, E., Ferraz-Mello, S. (eds.) Space Manifold Dynamics: Novel spaceways for science and exploration, pp. 97-112. Springer, New York (2010)

Guzzo, M., Lega, E., Froeschlé, C.: A numerical study of the topology of normally hyperbolic invariant manifolds supporting Arnold diffusion in quasi-integrable systems. Physica D 238, 1797-1807 (2009a)

Guzzo, M., Lega, E., Froeschlé, C.: A numerical study of Arnold diffusion in a priori unstable systems. Commun. Math. Phys. 290, 557-576 (2009b)

Hasselblatt, B., Pesin, Y.: Partially Hyperbolic Dynamical Systems. Handbook of Dynamical Systems, vol. 1B. pp. 1-55. Elsevier B. V., Amsterdam (2006)

Hirsch, M.W., Pugh, C.C., Shub, M.: Invariant Manifolds. Lecture Notes in Mathematics, vol. 583. Springer, Berlin (1977)

Lega, E., Guzzo, M., Froeschlé, C.: Detection of Arnold diffusion in Hamiltonian systems. Physica D 182, 179-187 (2003)

MacKay, R.S., Meiss, J.D., Percival, I.C.: Transport in Hamiltonian systems. Physica D 13, 55-81 (1984)

Melnikov, V.K.: On the stability of a center for time-periodic perturbations. Trudy Moskov. Mat. Obsc. 12, 3-52 (1963)

Poincaré, H.: Sur le problème des trois corps et les équations de la dynamique. Acta Mathematica 13, $1-271(1890)$

Simó, C.: On the analytical and numerical approximation of invariant manifolds. In: Benest, D., Froeschlé, Cl. (eds.) Modern Methods in Celestial Mechanics, pp. 285-329. Ed. Frontières, Gif-sur-Yvette (1989)

Treschev, D.: Trajectories in a neighborhood of asymptotic surfaces of a priori unstable Hamiltonian systems. Nonlinearity 15, 2033-2052 (2002)

Treschev, D.: Evolution of slow variables in a priori unstable Hamiltonian systems. Nonlinearity 17, 18031841 (2004)

Villac, B.F.: Using FLI maps for preliminary spacecraft trajectory design in multi-body environments. Celest. Mech. Dyn. Astron. 102, 29-48 (2008) 